\title{
Rheological Parameters of Shear-Thickening Fluids Using an Experimental Design
}

\author{
Alfredo Bosco $^{a} \mathbb{C}^{\mathbb{C}}$, Veronica Calado $^{a} * \mathbb{1}$, João Maia $^{b}$ \\ ${ }^{a}$ Universidade Federal do Rio de Janeiro, Av. Horácio Macedo, 2030, Cidade Universitária, 21941-914, \\ Rio de Janeiro, RJ, Brasil \\ ${ }^{b}$ Department of Macromolecular Science and Engineering, Case Western Reserve University, Cleveland, \\ $\mathrm{OH}, 44106$, USA
}

Received: September 21, 2018; Revised: August 07, 2019; Accepted: August 25, 2019

\begin{abstract}
The influence of important factors related to fluid preparation on shear-thickening fluids (STF) is not well explored in literature. Thus, this paper aimed at analyzing the statistical significance of experimental conditions, such as fumed silica concentration $\left(C_{s}\right)$, stirring time $\left(t_{s t}\right)$, stirring speed $\left(S_{s t}\right)$ and sonication time $\left(t_{s o}\right)$, on the rheological behavior of fumed silica particles and PEG 400 dispersions. For that, a $2^{\mathrm{k}}$ factorial Experimental Design (DOE), with three center points, was accomplished. The investigated response variables were: maximum viscosity $\left(\eta_{m}\right)$; critical shear rate $\left(\dot{\gamma}_{m}\right)$ where the viscosity is maximum; and agglomerate size of particles during preparation $(R)$. All factors were statistically significant for all three response variables. Higher viscosity values for shear rate range analyzed was obtained with longer $t_{s t}$, lower $S_{s t}$ and longer $t_{s o}$. An important contribution of this paper was to show discontinuous shear thickening (DST) for lower concentrations than previously found in literature only changing the preparation protocol.
\end{abstract}

Keywords: Rheology, Shear-thickening fluids, Fumed silica, Poly(ethylene glycol), Experimental design.

\section{Introduction}

Shear-thickening fluids can be defined as those whose viscosity increases as the shear rate increases. They are a type of non-Newtonian fluids and this specific rheological behavior presents a number of advantages for certain applications. In fact, Barnes ${ }^{1}$ states that shear-thickening behavior occurs in all suspensions, although this can often not be measured mechanically. In industrial scenarios, shear-thickening behavior may become a problem in view of mixer motor overloading; mixer blade damage; complications in pipe flow; and other losses $^{2,3}$. However, dispersions that present shear-thickening behavior have unique mechanical properties, which are useful in specific applications, such as liquid body armor, shock absorbers, packaging, and some others ${ }^{4,5}$.

The rheological behavior of fluids is controlled by a number of factors: suspension stability, particle size distribution, continuous phase viscosity, among others ${ }^{4}$. Presently, there are mainly two theories for describing the mechanism of shear-thickening behavior. The first one deals with the formation of so-called hydroclusters, according to which particle aggregation under shear promotes an increase in viscosity. The second mechanism predicts an increase in viscosity that derives from a change in a flow regime from ordered, layered flow, to disordered flow and this is often referred to as order-disorder transition.

The theory of hydroclusters has been introduced by Brady and Bossis ${ }^{6}$ and it is based on the concept that particles repel each other and move more independently under zero or low shear rates. This then results in lower viscosity values.
However, above a certain shear rate, particles assemble abruptly and temporarily in view of hydrodynamic forces. This mass of particles characterizes the formation of hydroclusters (Fig. 1) and the bigger these hydroclusters are, the higher the viscosity of the system is. As the shear rate increases, hydrocluster disruption eventually occurs. Another way that this may happen is when the shear stress is removed, as this allows for interparticle repulsion and a decrease in viscosity values ${ }^{8,9}$.

Figure 1a illustrates a slight change in the microstructure of a suspension at low shear rates, although Brownian motion is able to restore the initial configuration, which in turn results in a slight variation in viscosity values. As the shear rate increases, the structure loses its regeneration capacity and the microstructural changes compose small aggregates that orient in the flow direction. This process leads to a suspension with reduced viscosity and, thus, to a shear-thinning behavior (Fig. 1b). As the shear rate is increased further, hydrodynamic forces prevail in the system and its structure is drastically modified as a greater number of aggregates is formed. When the shear rate is greater than a critical value, small aggregates assemble in view of hydrodynamic forces and form hydroclusters. These hydroclusters act as barriers that contribute to a greater resistance to flow, i.e. a much higher viscosity (Fig. 1c) ${ }^{7}$.

Although the theory of hydroclusters formation is more broadly accepted to explain shear-thickening rheological behavior, the order-disorder transition mechanism predicts the similar shear rate values for the onset of shear-thickening behavior under the same conditions ${ }^{10}$. 
The order-disorder transition mechanism was developed by Hoffman ${ }^{11}$. According to the author, ordered packing of particles in a suspension with a high volume fraction would, above a given shear rate, break down into less-ordered arrangements. This would cause an increase in viscosity (Fig. 2).

However, it is worth mentioning that shear-thickening behavior is known to arise without the need for an orderdisorder transition mechanism to be present. Therefore, although order-disorder transition is a possible way that microstructural disorder coincides with shear-thickening behavior, it is not an indispensable mechanism for shearthickening behavior to take place ${ }^{2,12}$.

Several works from the literature work with suspensions of poly(ethylene glycol), PEG, and fumed silica in order to study their shear-thickening behavior. As indicated in Table 1, there is no pattern concerning the experimental conditions used. Thus, from recent data available in the literature, it is not possible to compare the influence of different factors on the rheological behavior of suspensions.
In order to do so, it is necessary to determine the concentration and suitable preparation method in order to obtain stable suspensions and reliable results. Great care must be taken while stirring the suspensions during their preparation, because stirring may itself cause some degree of shearing and interfere significantly in the rheological behavior of suspensions. PEG is a good candidate as a continuous phase for shear-thickening fluids that contain fumed silica as the disperse phase. The reason for this is that PEG has high thermal stability; low volatility; and a refraction index that is similar to that of fumed silica. Therefore, all these factors contribute to suspension stability ${ }^{20}$.

The present work deals with the rheological behavior of suspensions composed of PEG and fumed silica. In this sense, the objective was to verify the influence of four factors on the following response variables: maximum viscosity, critical shear rate, and agglomerate size in suspension. This was carried out based on a $2^{4}$ Factorial Experiment Design, whose factors were: fumed silica concentration $\left(C_{s t}\right)$, stirring time $\left(t_{s t}\right)$, stirring speed $\left(S_{s t}\right)$, and sonication time $\left(t_{s o}\right)$. (a)

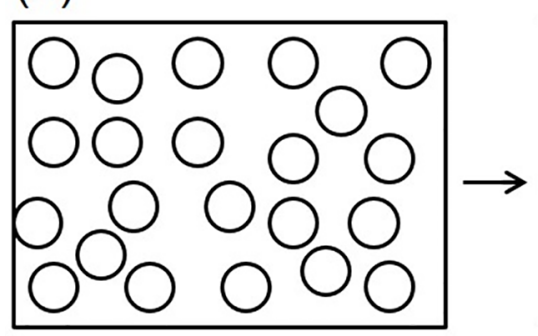

(b)

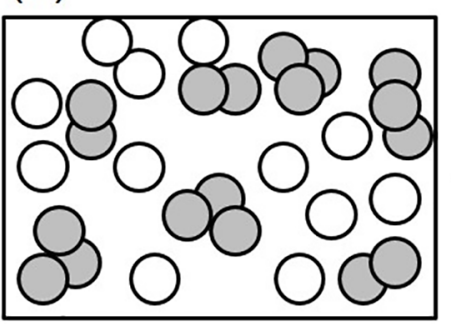

(c)

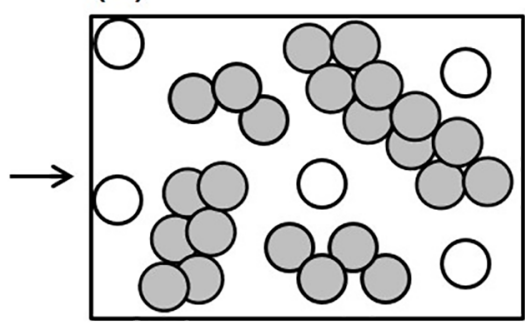

\section{shear rate}

Figure 1. Scheme illustrating hydroclusters formation. Adapted from Liu et al.
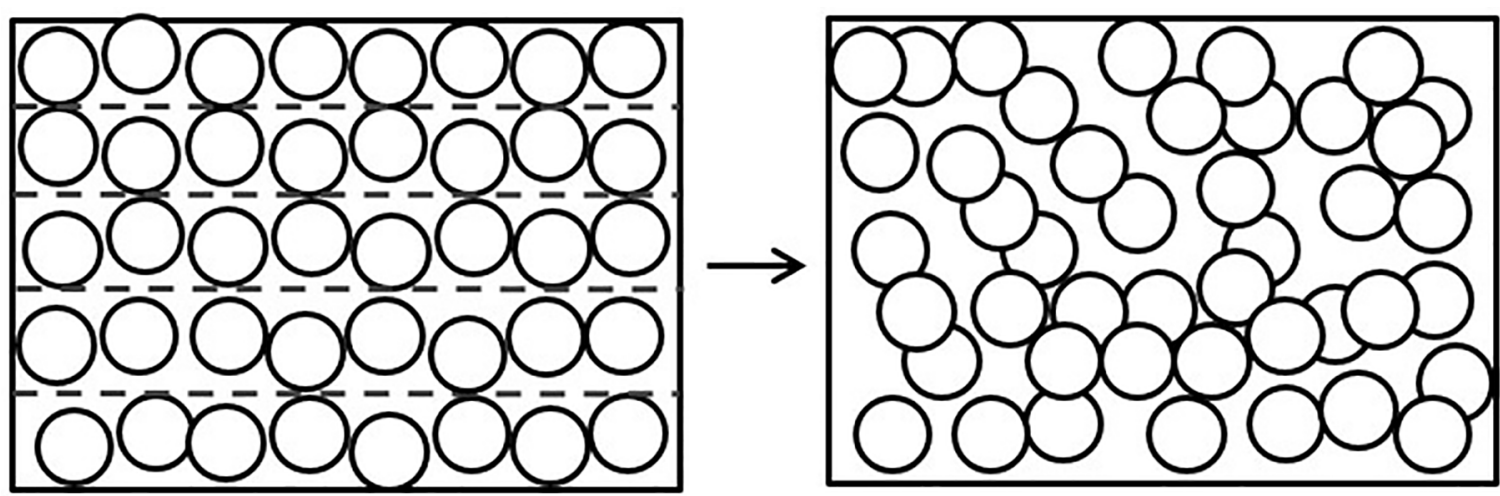

\section{shear rate}

Figure 2. Scheme illustrating mechanism of order-disorder transition. 


\section{Materials and Methods}

Poly(ethylene glycol), PEG, with a molar mass of $400 \mathrm{~g}$. $\mathrm{mol}^{-1}$, was purchased from Vetec Química Fina Ltda (Brazil) and used as the continuous phase. Fumed silica nanoparticles $\left(\right.$ Aerosil $\left.{ }^{\circledR} 200\right)$, with a specific surface area of $200 \mathrm{~m}^{2} \cdot \mathrm{g}^{-1}$, were kindly donated by Evonik Industries AG (Germany) and used as the disperse phase. Aerosil ${ }^{\circledR} 200$ are synthetic silicone dioxide nanoparticles, which can be obtained via a high-temperature reaction that involves silicon tetrachloride, hydrogen, and oxygen:

$$
\mathrm{SiCl}_{4}+2 \mathrm{H}_{2}+\mathrm{O}_{2} \rightarrow \mathrm{SiO}_{2}+4 \mathrm{HCl}
$$

Fumed silica is a non-porous, amorphous substance with a high degree of purity ( $>99.9 \%$ ) and finds its main application in silicon composite manufacturing ${ }^{10}$. It provides the final composite product characteristics such as high resistance to oxidation, good chemical inertia, low dielectric constant, and high reinforcement effect.

A Factorial $2^{4}$ Experimental Design was carried out with three center point replicates adding up to a total of 19 experiments ${ }^{13}$. The aim was at identifying which factors were important when studying the rheological behavior of these suspensions, as well as which factor levels were more adequate to prepare a suspension with a higher degree of shear-thickening behavior.
It is worth mentioning that the existence of center points allows one to evaluate the plane curvature statistical significance. Factors and factor levels chosen for this paper, which were based on the works presented in Table 1, are listed in Table 2. Statistica software, version 13 from StatSoft ${ }^{\mathbb{R}}$, was used to generate the design matrix and to analyze experimental data. No prediction model will be presented here, as the only objective was to detect the statistical significance of the factors.

Fumed silica nanoparticles were suspended in PEG 400 and stirred under vacuum in a Dispermat ${ }^{\circledR}$ LC 30 (VMAGetzmann) instrument in order to promote a homogeneous suspension. Samples were then submitted to sonication on a Soni-Tech 2510R-MTH $42 \mathrm{kHz}$ (Branson) sonicator to increase the degree of dispersion of the colloidal particles and reduce the degree of particle aggregation. Subsequently, samples were kept at rest during $24 \mathrm{~h}$ at room temperature to remove as much as possible air bubbles that may have formed during the preparation procedure (after sonication).

Suspension characterization was carried out using a Zetasizer $^{\circledR}$ ZEN 3600 (Malvern) particle analyzer, which was also used to determine the particle size in suspension. A Turbiscan LAB (Dafratec) was used to analyze the suspension stability. Rheological measurements in steady-state were obtained at $25^{\circ} \mathrm{C}$ on an ARG2 (TA Instruments) rheometer, fitted with a $40 \mathrm{~mm}, 2^{\circ}$ cone-and-plate geometry, gap at 100 $\mu \mathrm{m}$. The herein analyzed shear rate range was from 0.1 to $1000 \mathrm{~s}^{-1}$, with one point at every $0.5 \mathrm{~s}$.

Table 1. Rheological studies of silica-poly(ethylene glycol) suspensions.

\begin{tabular}{|c|c|c|c|c|c|c|}
\hline Authors & $\begin{array}{c}\text { Fumed silica } \\
\text { concentration - } \\
\text { Continuous phase }\end{array}$ & $\begin{array}{l}\text { Stirring } \\
\text { time }\end{array}$ & $\begin{array}{l}\text { Stirring } \\
\text { speed }\end{array}$ & Sonication time & $\begin{array}{l}\text { Critical shear rate } \\
\qquad\left(\mathrm{s}^{-1}\right)\end{array}$ & $\begin{array}{c}\text { Maximum } \\
\text { viscosity }^{2}(\mathrm{~Pa} \cdot \mathrm{s})\end{array}$ \\
\hline Wu et al. $(2006)^{14}$ & $9 \%$ - PEG 200 & $30 \mathrm{~min}$ & $\begin{array}{c}\text { Non } \\
\text { informed }\end{array}$ & $0 \mathrm{~h}$ & 155 & 1,5 \\
\hline Ozel et al. $(2014)^{10}$ & $20 \%$ - PEG 200 & $4 \mathrm{~h}$ & $\begin{array}{c}5000 \\
\mathrm{rpm}\end{array}$ & $0 \mathrm{~h}$ & 130 & 11 \\
\hline Liu et al. $(2015)^{7}$ & $20 \%$ - PEG 400 & $8 \mathrm{~h}$ & $\begin{array}{l}\text { Non } \\
\text { informed }\end{array}$ & $2 \mathrm{~h}$ & 70 & 50 \\
\hline Haris et al. $(2015)^{15}$ & $27.5 \%$ - PEG 200 & $\begin{array}{l}\text { from } 30 \\
\text { to } 90 \mathrm{~min}\end{array}$ & $\begin{array}{l}\text { Non } \\
\text { informed }\end{array}$ & $\begin{array}{l}\text { from } 30 \text { min to } \\
\text { several hours }\end{array}$ & 1000 & 20 \\
\hline Kang et al. $(2012)^{16}$ & $27.5 \%$ - PEG 200 & $1 \mathrm{~h}$ & $\begin{array}{l}\text { Non } \\
\text { informed }\end{array}$ & $10 \mathrm{~h}$ & 825 & 3 \\
\hline Fahool and Sabet $(2015)^{17}$ & $35 \%$ - PEG 400 & $2 \mathrm{~h}$ & $\begin{array}{l}1000 \\
\mathrm{rpm}\end{array}$ & $10 \mathrm{~min}$ & 60 & 550 \\
\hline Liu et al. $(2015)^{7}$ & $40 \%$ - PEG 200 & $8 \mathrm{~h}$ & $\begin{array}{c}\text { Non } \\
\text { informed }\end{array}$ & $2 \mathrm{~h}$ & 500 & 80 \\
\hline Hasanzadeh et al. $(2015)^{18}$ & $45 \%$ - PEG 200 & $\begin{array}{c}\text { Non } \\
\text { informed }\end{array}$ & $\begin{array}{l}3000 \\
\mathrm{rpm}\end{array}$ & $0 \mathrm{~h}$ & 80 & 440 \\
\hline
\end{tabular}

${ }^{1}$ Critical shear rate: the point where there is a transition between shear-thickening and shear-thinning behaviors. ${ }^{2}$ Maximum viscosity: Observed at a critical shear rate

Table 2. Factors and factor levels

\begin{tabular}{cccc}
\hline Factors & Low level & Center point & High level \\
\hline Fumed silica concentration (\% in volume),Cst & 5 & 15 & 20 \\
Stirring time (h), tst & 0.5 & 4.25 & 8 \\
Stirring speed (rpm),Sst & 300 & 1650 & 3000 \\
Sonication time (h), so & 0 & 1 & 2 \\
\hline
\end{tabular}




\section{Results and Discussion}

SEM and TEM micrographs of fumed silica are shown in Fig. 3; it is composed of highly anisotropic, randomly organized aggregates of $200 \mathrm{~nm}$ to $500 \mathrm{~nm}$ in length that is in turn comprised of primary particles with a diameter from $10 \mathrm{~nm}$ to $20 \mathrm{~nm}$; this is in accordance with the previous studies ${ }^{9,10,19}$.

\subsection{Statistical analysis}

The effects of the following factors were evaluated, all relating to the preparation of shear-thickening fluids: fumed silica concentration, stirring time, stirring speed, and sonication time. Results pertaining to each experimental condition are presented in Table 3.

Statistical conclusions presented in this work are associated with a $5 \%$ level of significance (p-level).

For maximum viscosity, $\eta_{m}, C_{s}$ was the most statistically relevant factor of all. This is expected because suspension viscosity is often related to solids concentration in a direct manner. Negative values for the effects of $S_{s t}$ and $t_{s t}$ are also expected as higher $S_{s t}$ and $t_{s t}$ factor levels yield lower viscosity values.

Sonication time, $t_{s o}$, was the only statistically nonsignificant factor in determining $\eta_{m}$, but its interactions with $t_{s t}$ and $C_{s}$ were statistically significant for $\eta_{m}$. This implies that factor $t_{s o}$ should not be disregarded. In other words, all factors under investigation influenced $\eta_{m}$.
For critical shear rate, $\dot{\gamma}_{m}$, the statistical significance of factor fumed silica concentration $C_{s}$ evidently stood out compared to other factors and their respective interactions. Therefore, $C_{s}$ was the factor responsible for determining critical shear rate $\dot{\gamma}_{m}$. Although sonication time, $t_{s o}$, did not exhibit statistical significance, its interaction terms did. Consequently, this factor was relevant and affected the critical shear rate $\dot{\gamma}_{m}$. The same conclusion applied to stirring time, tst. Moreover, as expected, higher particle concentrations in suspensions demanded lower shear rate values in order to reach a maximum viscosity, $\eta_{m}$. This was reflected by the negative effect of factor $\mathrm{C}_{\mathrm{s}}$ on the critical shear rate $\dot{\gamma}_{m}$.

For agglomerate size, $R, C_{s}$, and $S_{s t}$ were statistically significant, with $\mathrm{C}_{\mathrm{s}}$ being more statistically significant than $S_{s t}$. Stirring time, $t_{s}$, and sonication time, $t_{s o}$, were statistically nonsignificant in determining agglomerate size, $R$. The interaction factor between $C_{s}$ and $t_{s o}$ factors was statistically significant for the agglomerate size, $R$, similarly to trends observed previously, implying that factor $t_{\text {so }}$ should not be disregarded.

Figures 4-6 present means plots that allow to easily figure out trends relating response variables and factors. Because it is not possible to evaluate more than 3 factors in a single means plot and there are four factors, $\mathrm{C}_{\mathrm{s}}$ was left out of the graphs (its value was fixed at the center point, 12.5\%) as it was statistically significant for all three response variables. The average center point $(+)$ of the four factors was placed on the graphs.

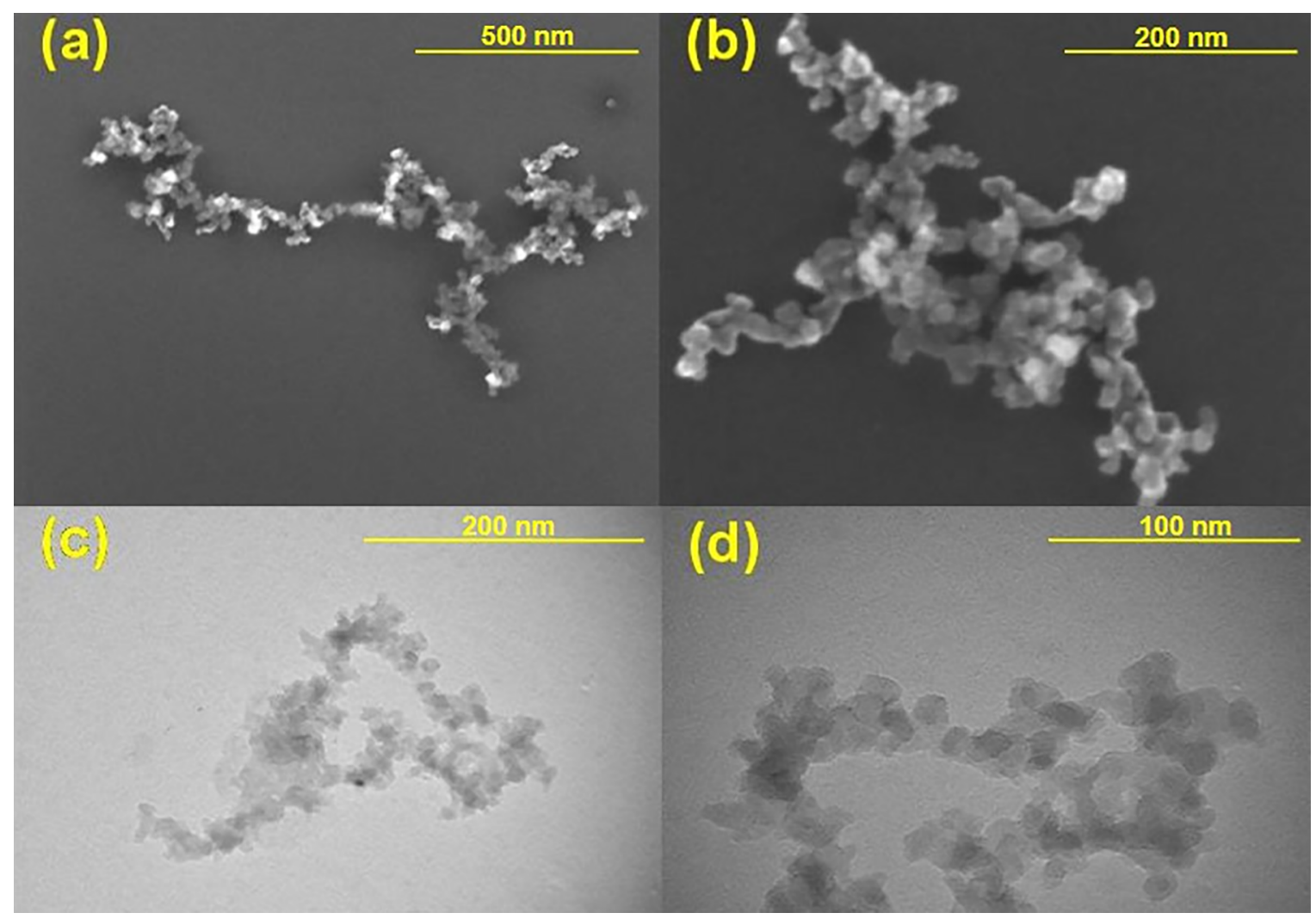

Figure 3. SEM micrographs (a) and (b); and TEM micrographs (c) and (d) of fumed silica nanoparticles. 
Table 3. Experimental design matrix with factors, their levels, and response variable values

\begin{tabular}{|c|c|c|c|c|c|c|c|}
\hline Sample & $\begin{array}{c}\text { Fumed silica } \\
\text { concentration, Cs }(\%)\end{array}$ & $\begin{array}{c}\text { Stirring time, } \\
t_{s t}(\mathrm{~h})\end{array}$ & $\begin{array}{l}\text { Stirring speed, } \\
S_{s t}(\mathrm{rpm})\end{array}$ & $\begin{array}{c}\text { Sonication time, } \\
t_{s o}(\mathrm{~h})\end{array}$ & $\begin{array}{c}\text { Maximum viscosity, } \\
\eta_{m}(\mathrm{~Pa} \cdot \mathrm{s})\end{array}$ & $\begin{array}{l}\text { Critical shear } \\
\text { rate } \dot{\gamma}_{m}\left(\mathrm{~s}^{-1}\right)\end{array}$ & $\begin{array}{l}\text { Agglomerate } \\
\text { size } R(\mathrm{~nm})\end{array}$ \\
\hline 1 & 5 & 8 & 3000 & 2 & a & $\mathrm{a}$ & 881 \\
\hline 2 & 20 & 8 & 3000 & 2 & 140 & 34.49 & 2416 \\
\hline 3 & 20 & 8 & 300 & 0 & 124.7 & 38.51 & 2520 \\
\hline 4 & 5 & 8 & 300 & 0 & 0.9 & 277.1 & 1911 \\
\hline 5 & 12.5 & 4.25 & 1650 & 1 & 17.7 & 292 & 2461 \\
\hline 6 & 5 & 8 & 3000 & 0 & a & a & 1167 \\
\hline 7 & 20 & 0.5 & 300 & 0 & 150 & 36 & 2492 \\
\hline 8 & 5 & 0.5 & 3000 & 0 & a & a & 732 \\
\hline 9 & 5 & 0.5 & 3000 & 2 & 1.15 & 292.3 & 813 \\
\hline 10 & 5 & 0.5 & 300 & 2 & a & a & 1706 \\
\hline 11 & 12.5 & 4.25 & 1650 & 1 & 15.8 & 294 & 2317 \\
\hline 12 & 5 & 8 & 300 & 2 & 2.35 & 289.2 & 1449 \\
\hline 13 & 5 & 0.5 & 300 & 0 & a & a & 1844 \\
\hline 14 & 20 & 8 & 3000 & 0 & 77.45 & 47.75 & 2001 \\
\hline 15 & 20 & 0.5 & 3000 & 2 & 62.47 & 52.57 & 2330 \\
\hline 16 & 12.5 & 4.25 & 1650 & 1 & 14.6 & 291.7 & 2453 \\
\hline 17 & 20 & 0.5 & 300 & 2 & 57.93 & 58.58 & $\mathrm{~b}$ \\
\hline 18 & 20 & 8 & 300 & 2 & 123.7 & 39.72 & b \\
\hline 19 & 20 & 0.5 & 3000 & 0 & 61.92 & 58.6 & 1970 \\
\hline
\end{tabular}

${ }^{\mathrm{a}}$ They did not present shear-thickening behavior. Instead, they presented a shear-thinning or nearly Newtonian behavior. ${ }^{\mathrm{b}}$

The suspension could not be poured into the particle analyzer's cuvette to determine agglomerate size because its consistency.

There are two sets of conditions, statistically similar, that lead to higher $\eta_{m}$ values, as can be seen in Fig. 4. However, one of them is more energy and time demanding $\left(C_{s}=12.5 \%\right.$, $t_{s t}=8 \mathrm{~h}, S_{s t}=3000 \mathrm{rpm}$ and $t_{s o}=2 \mathrm{~h}$ ). Consequently, it is suggested that the other set of conditions should be used for obtaining higher maximum viscosity, $\eta_{m}\left(C_{s}=12.5 \%, t_{s t}=\right.$ $0.5 \mathrm{~h}, S_{s t}=300 \mathrm{rpm}$ and $t_{s o}=0 \mathrm{~h}$ ).

From the means plot in Fig. 4, it is possible to infer that there is a point of minimum, as the center point corresponds to $16 \mathrm{~Pa} \cdot \mathrm{s}$ (represented by + ) for maximum viscosity, $\eta_{m}$, the lowest value of all experiments.

In terms of the response variable critical shear rate, $\dot{\gamma}_{m}$, on the other hand, it is possible that there exists a point of maximum in view of a center point average of $293 \mathrm{~s}^{-1}$ (Fig. 5).

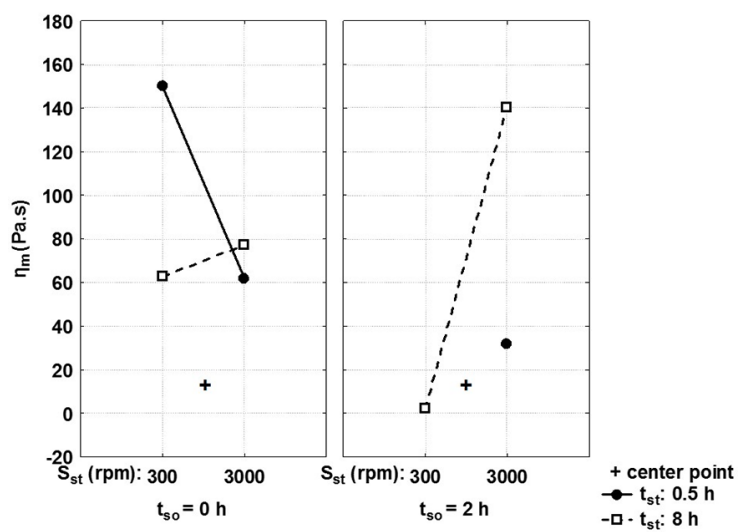

Figure 4. Means plot for maximum viscosity $\eta m$. Symbols (+) are related to the center point average.

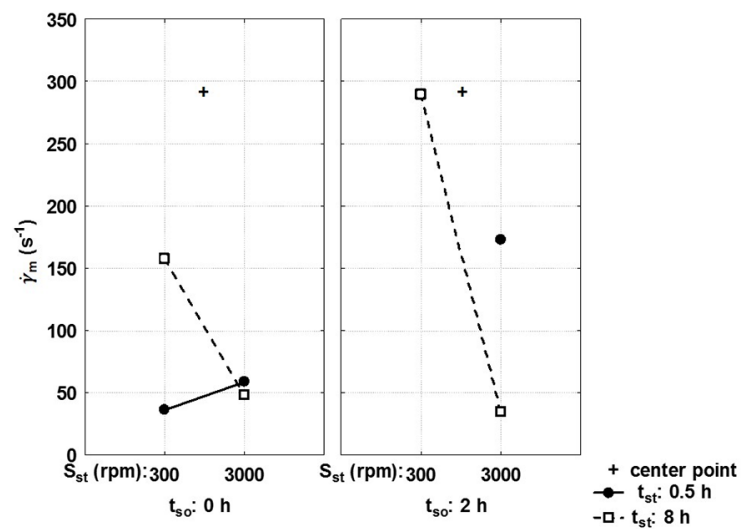

Figure 5. Means plot for maximum critical shear rate $\dot{\gamma}_{m}$. Symbols $(+)$ are related to center point average.

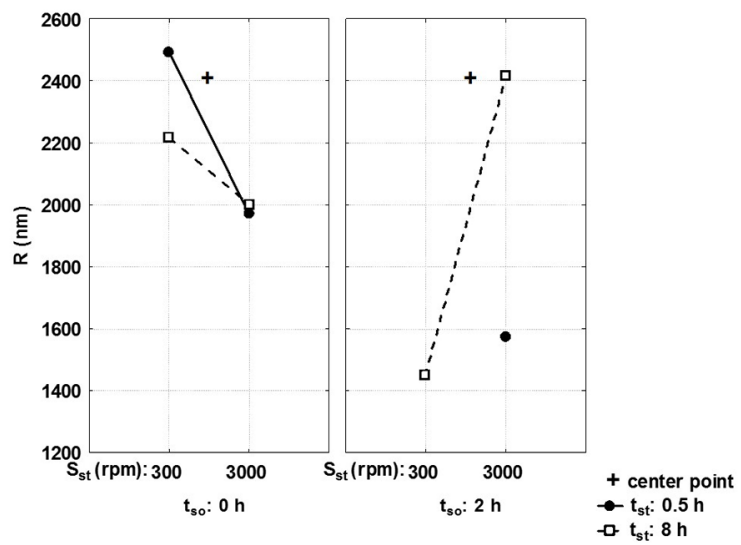

Figure 6. Means plot agglomerate size $(R)$. Symbols $(+)$ are related to center point average. 
In order to obtain higher $\dot{\gamma}_{m}$, the set of conditions to be used is: $C_{s}=12.5 \%, t_{s t}=8 \mathrm{~h}, S_{s t}=300 \mathrm{rpm}$ and $t_{s o}=2 \mathrm{~h}$.

As occurred for maximum viscosity, $\eta_{m}$, there are two sets of conditions that lead to higher agglomerate size $R$ values (Fig. 6), and one of them is more energy and time demanding $\left(C_{s}=12.5 \%, t_{s t}=8 \mathrm{~h}, S_{s t}=300 \mathrm{rpm}\right.$ and $t_{s o}=$ $2 \mathrm{~h}$ ). Therefore, the other set should be used ( $C_{s}=12.5 \%$, $t_{s t}=0.5 \mathrm{~h}, S_{s t}=300 \mathrm{rpm}$ and $t_{s o}=0 \mathrm{~h}$ ). It is interesting to obtain high agglomerate size $R$ values because agglomerates contribute for suspensions with high viscosities.

For maximum viscosity, $\eta_{m}, C_{s}$ and $S_{s t}$ were the most statistically significant factors, with a positive value of the effect for $C_{s}$ and negative value of the effect for $S_{s t}$. For higher $C_{s}(20 \%)$ and lower $S_{s t}(300 \mathrm{rpm}), \eta_{m}$ increases with decreasing $t_{s t}$ and $t_{s o}$. Curvatures presented in contour lines indicate an interaction between $t_{s t}$ and $t_{s o}$ (Fig. 7), considering all samples.

\subsection{Rheological behavior}

Initially, suspensions present a shear-thinning rheological behavior at low shear rates and all flow curves are shown in Figure 8. However, from a given value of shear rate (onset shear rate) onward, suspensions present shear-thickening behavior in view of suspension microstructure alterations. This onset shear rate value varies with different factor levels used. Shear-thickening behavior is maintained up to a critical shear rate is achieved. From this point onwards, shear-thinning behavior is present again.

As discussed in the Introduction, there are mainly two possible theories to describe shear-thickening behavior of suspensions: order-disorder transition mechanism and hydroclusters formation. The order-disorder transition mechanism is not applicable to the present study because particles must have some degree of order before shearthickening behavior is observed and herein analyzed fumed silica particles do not have a regular shape.
Therefore, this does not allow for ordered structures to form. On the other hand, hydroclusters theory is more broadly accepted and more suitable to describe the system under investigation. Additionally, hydroxyl groups that are present at the ends of PEG chains are able to hydrogen bond with silanol groups on the surface of particles ${ }^{18}$. This also favors hydrocluster formation and leads to shear-thickening rheological behavior.

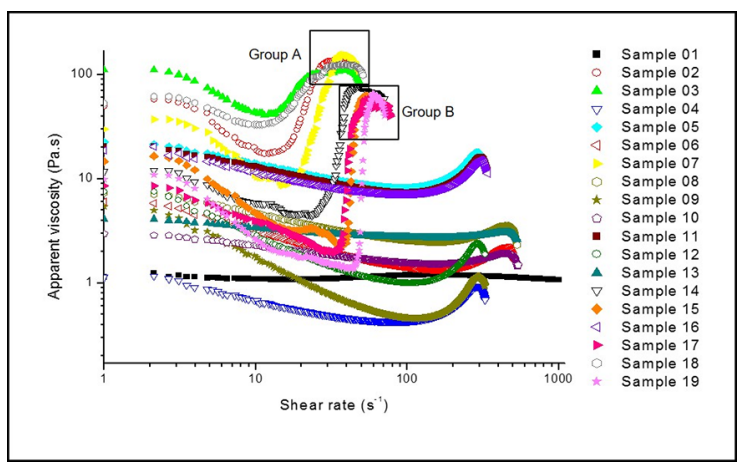

Figure 8. Apparent viscosity versus shear rate for silica-PEG 400 suspensions.

Suspension samples 17 and 18 presented yield stress, which did not allow for pouring into the particle analyzer's cuvette to determine agglomerate size. These samples were prepared with a higher $C_{s}$ level $(20 \%)$; lower $S_{s t}$ level (300 $\mathrm{rpm})$; and higher $t_{s o}$ level $(2 \mathrm{~h})$, that can lead to, respectively, an increase in yield stress; less intensive shearing; and improved particle distribution. These conditions would contribute to fluid structuring and cause an increase in yield stress.

As discussed in the Statistical Analysis section, Cs was by far the most relevant factor for all response variables. Therefore, samples and trends in Fig. 9 can be analyzed in groups according to $C_{s}$ factor levels.

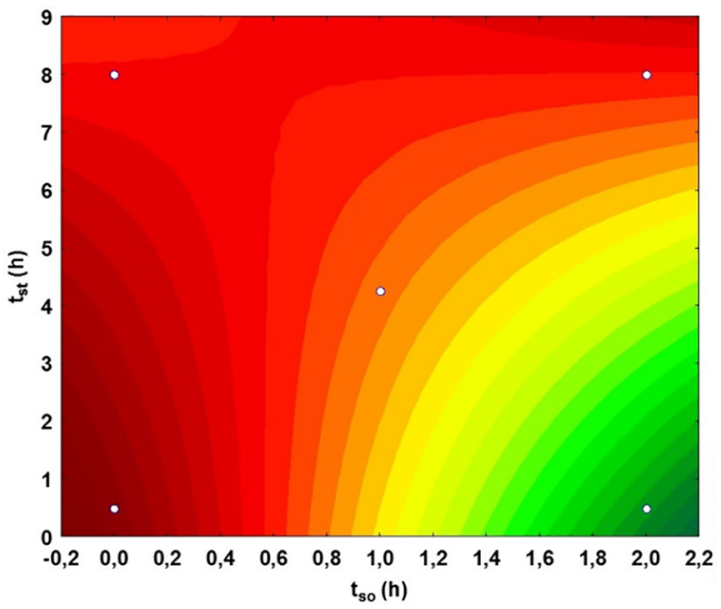

Figure 7. Effect of maximum viscosity $\left(\eta_{m}\right)$ with $C_{s}=20 \%$ in volume (silica concentration) and $S_{s t}=300 \mathrm{rpm}$ (stirring speed). 


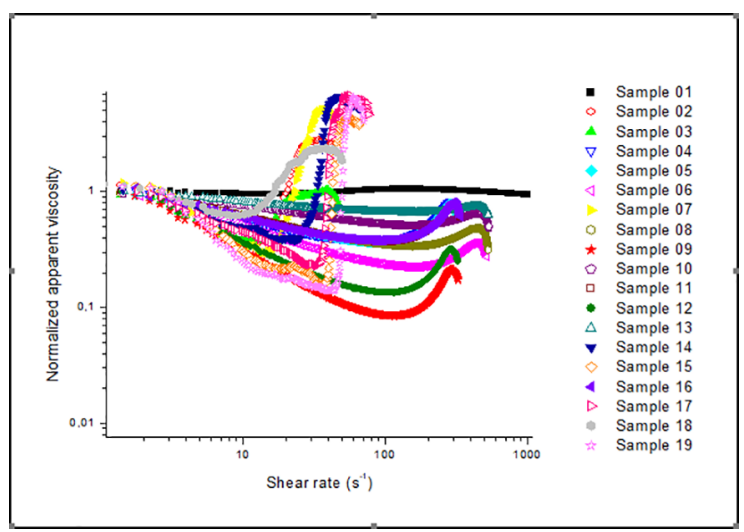

Figure 9. Normalized apparent viscosity as a function of shear rate.

Samples with $C_{s}=20 \%$ presented higher $\eta_{m}$ and were then subdivided into two groups: group $\mathrm{A}$ is composed of samples 02, 03, 07 and 18; and group B comprises samples 14, 15, 17 and 19. Group A samples presented higher $\eta_{m}$ and lower $\dot{\gamma}_{m}$ than the ones from group B. As illustrated in Table $3, \eta_{m}$ values from group A samples varied from 123.7 to $150 \mathrm{~Pa} \cdot \mathrm{s}$ and $\dot{\gamma}_{m}$ values were in the range of 34.49 to $39.72 \mathrm{~s}^{-1}$; for group B, these ranges varied from 57.93 to $77.45 \mathrm{~Pa} \cdot \mathrm{s}$ and from 47.75 to $58.6 \mathrm{~s}^{-1}$. Maximum viscosities values obtained in this work, for fumed silica concentration of $20 \%$, were higher than those obtained by Ozel et al..$^{10}$ and by Liu et al. ${ }^{7}$, i.e. 11 and $50 \mathrm{~Pa} \cdot \mathrm{s}$, respectively, as presented in Table 1.

Samples belonging to group A showed a continuous shearthickening behavior, while samples belonging to group $B$ showed a discontinuous shear-thickening behavior, in accordance with the results presented by Jamali et al. ${ }^{9}$ and Jiang and Zukoski ${ }^{21}$.

Continuous/discontinuous shear-thickening behavior was probably related to the packing of agglomerates generated during sample preparation. In view of an irregular morphological distribution of fumed silica particles as received, they could be agglomerated because of physical entanglements between themselves. With a lower stirring time $\left(t_{s t}=0.5 \mathrm{~h}\right)$, these physical entanglements would remain in suspension even after samples had been prepared and would thus form suspensions with less packable agglomerates (group B). As to a higher stirring time $\left(t_{s t}=8 \mathrm{~h}\right.$ ), these physical entanglements would not only be undone but also fumed silica particles would rupture as a result of the action of the mixer. Thus, suspensions with agglomerates that are less physically entangled, i.e., more packable (group A) would form. Hydrodynamic forces would prevail in the formation of group A agglomerates, while physical entanglements would prevail in the formation of agglomerates related to group $B$.

Available literature on shear thickening fluids using fumed silica has no concern about agglomeration that fumed silica presents when received from the supplier. According to this present work, the degree of agglomeration clearly influences the results obtained. Therefore, degree of agglomeration analysis is fundamental to obtain reliable results.

Simultaneous, clearer visualization of rheological behavior of several samples is made possible by normalized apparent viscosity. All values of apparent viscosity were divided by their respective initial value. This strategy disregards the initial state of each sample and allows for identification of specific trends and relative comparisons; besides, it highlights the formation kinetics of hydroclusters, as shown in Fig. 9.

Higher degree of packing would contribute to greater interaction between group A agglomerates in comparison with those of group B and lower shear rates would be required for hydrocluster formation. Samples belonging to group A indeed presented lower critical shear rates than samples from group B, as shown in Figures 8 and 9.

Trends in apparent viscosity would also be related to hydrocluster packing. Less packed hydroclusters would allow suspensions to reach higher viscosities at higher shear rates, as occurred in group B samples. Suspensions formed by more packed hydroclusters reached higher viscosities at lower shear rates, as occurred in group A samples.

The hydroclusters formation rate is proportional to the slope of flow curves during the shear-thickening behavior. This slope is related to the flow behavior index $(n)$ presented in the Ostwald de Waele model, or Power Law:

$$
\eta=\mathrm{K} \dot{\gamma}_{m}^{(n-1)}
$$

where $\eta$ is the apparent viscosity; $\mathrm{K}$ is the flow consistency index; and $\dot{\gamma}$ is the shear rate. For shear-thickening behavior of samples with $C s=20 \%$, the flow behavior indexes are presented in Table 4.

Table 4. Flow behavior indexes for shear-thickening behavior of samples with $20 \%$ fumed silica concentration. SD = standard deviation and $\mathrm{R}^{2}=$ coefficient of determination

\begin{tabular}{cccc}
\hline Group sample & Samples & $\boldsymbol{n} \pm \mathrm{SD}$ & $\mathrm{R}^{2}$ \\
\hline A & 18 & $2.75 \pm 0.05$ & 0.99 \\
A & 03 & $2.75 \pm 0.06$ & 0.91 \\
A & 07 & $4.37 \pm 0.18$ & 0.96 \\
A & 02 & $4.59 \pm 0.10$ & 0.99 \\
B & 14 & $9.77 \pm 0.40$ & 0.98 \\
B & 19 & $9.94 \pm 0.82$ & 0.90 \\
B & 17 & $12.33 \pm 1.02$ & 0.94 \\
B & 15 & $14.94 \pm 0.83$ & 0.97 \\
\hline
\end{tabular}


Higher $n$ values were observed in group B samples and, thus, characterize discontinuous shear-thickening behavior, which would have been mainly owing to physical entanglements between agglomerates to form hydroclusters. On the other hand, lower $n$ values were observed in group A samples, which characterizes continuous shear-thickening behavior, which would have been in turn mainly on account of the formation of hydroclusters by the hydrodynamic forces.

According to Barnes ${ }^{1}$, hydroclusters are broken down at high shear rates when they are formed by hard particles. Hence, this reduces apparent viscosity. However, for the case of soft particles, hydroclusters are not undone but rather deformed in the direction of flow at high shear rates. In this case, apparent viscosity also decreases (Fig. 10). For a suspension formed by soft particles, the critical shear rate tends to be lower than the one from suspension formed by hard particles. The reason for this is that less energy would be required to deform packable agglomerates than to undo agglomerates with hard particles.

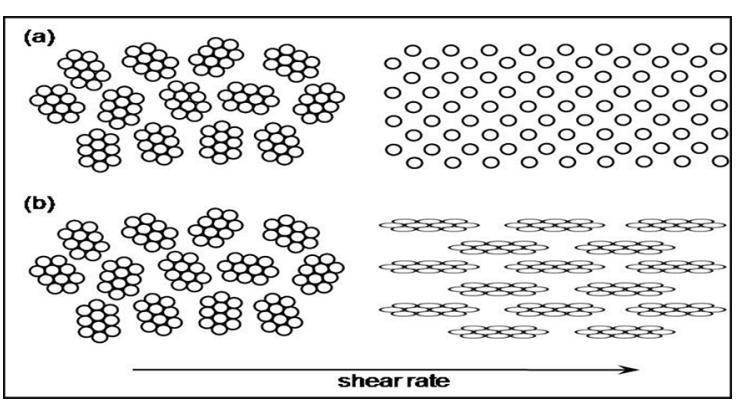

Figure 10. Breaking down of hydroclusters formed by hard particles (a) and deformation of hydroclusters formed by soft particles (b) as the shear rate is increased.

Agglomerates in suspensions probably presented different degrees of packing given the presence of free volume in their interiors. In much the same way that agglomerates with lower degrees of packing could be treated analogously as hard particles, those with higher degrees of packing could be treated analogously as soft particles.

Samples with $C_{s}=5 \%$ did not show shear-thickening behavior so expressive as that of samples with $C_{s}=20 \%$. Preparation of samples 04 and 12 differed only with respect to $t_{\text {so }}$ : sample 12 had been submitted to sonication treatment $\left(t_{s o}=2 \mathrm{~h}\right)$ and sample 04 had not. It appears that sonication treatment favored particle distribution and decreased the degree of agglomeration. This would cause a decrease in agglomerate size $(R)$ values and would reflect a lower viscosity for sample 12 in the entire range of shear rates studied, as shown in Fig. 9. This same principle could be applied when comparing samples 13 and 10 .

Samples 09 and 12 were prepared with different $t_{s t}$ (stirring time) and $S_{s t}$ (stirring speed). According to ANOVA test for $\eta_{m}, S_{s t}$ presented higher statistical significance than $\mathrm{t}_{\mathrm{st}}$. In other words, $S_{s t}$ prevailed over $t_{s t}$.
Higher $S_{s t}$ during the preparation of sample 09 may have promoted some degree of damage in suspension microstructure, causing a decrease in viscosity compared to sample 12 (Fig. 9). Again, according to ANOVA test (p-level ( 0.05) for $R, S_{s t}$ was statistically significant while $t_{s t}$ did not present statistical significance. It is possible that a higher $S_{s t}$ level may have compromised the interactions between particles and may have made it difficult for agglomerates to form. This could explain smaller agglomerate sizes for this sample in comparison with sample 12 . This same principle could be applied when comparing samples 06 and 13 .

It is not in all cases that an increase in shear rate causes hydrocluster formation and results in an increase in viscosity. Flow curves for samples 06, 08, 10 and 13 showed similar behavior in the analyzed shear rate range. Viscosity values for these samples did not undergo a significant increase and decreased abruptly when a shear rate of $465 \mathrm{~s}^{-1}$ was reached. This was possibly due to structural disassemble/orientation in the fluid microstructure during shearing.

For $C_{s}=5 \%$ and $t_{s t}=0.5 \mathrm{~h}$, samples 08 and 10 differed between each other in $S_{s t}$ (3000 rpm and $300 \mathrm{rpm}$, respectively) and $t_{s o}$ (respectively, no sonication treatment and $t_{s o}=2 \mathrm{~h}$ ). $S_{s t}$ was responsible for the difference between these samples, because it was a factor more statistically significant than $t_{s o}$, as shown by ANOVA test. A higher $S_{s t}$ resulted in lower agglomerate size $(R)$ for sample 08 than for sample 10 (Table 3 ). Higher $S_{s t}$ also disturbed the suspension microstructure to a greater extent and caused sample 08 to be less viscous than sample 10 in the entire shear rate range (Fig. 9).

Microstructural disassemble may explain the fact that sample 01 presented roughly Newtonian behavior as this sample underwent longer $t_{s t}(8 \mathrm{~h})$, higher $S_{s t}(3000 \mathrm{rpm})$ and longer $t_{s o}(2 \mathrm{~h})$.

\section{Conclusions}

In order to have a higher viscosity in a higher critical shear rate, samples need to be prepared with higher fumed silica concentration, longer stirring time, lower stirring speed and longer sonication time.

Because we are interested in the shear-thickening behavior, samples 02 (fumed silica concentration of $20 \%$, stirring time of $8 \mathrm{~h}$, stirring speed of $3000 \mathrm{rpm}$ and sonication time of $2 \mathrm{~h}$ ) and 07 (fumed silica concentration of $20 \%$, stirring time of $0.5 \mathrm{~h}$, stirring speed of $300 \mathrm{rpm}$ and no sonication) would be the most promising options because they resulted the highest maximum viscosities. From this perspective, sample 02 would be even more appropriate because it presents higher viscosity values for the most part of the shear rate range. In terms of shear rate range, sample 03 would be more suitable for future work because presented the highest viscosities in almost all its shear rate range studied (from 0.1 to $20 \mathrm{~s}^{-1}$ ), even though its maximum viscosity value was lower than that of the aforementioned samples. 


\section{Acknowledgment}

The authors would like to thank CAPES for the fellowship of the first author and also Evonik Industries AG for the donation of Aerosil ${ }^{\circledR} 200$ fumed silica nanoparticles.

\section{References}

1. Barnes HA. A handbook of elementary rheology. Aberystwyth: Cambrian Printers; 2000.

2. Egres RG, Wagner NJ. The rheology and microstructure of acicular precipitated calcium carbonate colloidal suspensions through the shear thickening transition. Journal of Rheology. 2005;49(3):719-746.

3. Jiang W, Sun Y, Xu Y, Peng C, Gong X, Zhang Z. Shear-thickening behavior of polymethylmethacrylate particles suspensions in glycerine-water mixtures. Rheologica Acta. 2010;49:1157-1163.

4. Mewis J, Wagner NJ. Colloidal suspension rheology. Cambridge: University Press Cambridge; 2012.

5. Ding J, Tian T, Meng Q, Guo Z, Li W, Zhang P, Ciacchi FT, Huang J, Yang W. Smart Multifunctional Fluids for Lithium Ion Batteries: Enhanced Rate Performance and Intrinsic Mechanical Protection. Scientific Reports. 2013;3:2485.

6. Brady JF, Bossis G. The rheology of concentrated suspensions of spheres in simple shear-flow by numerical-simulation. Journal of Fluid Mechanics. 1985;155:105-129.

7. Liu XQ, Bao RY, Wu XJ, Yang W, Xie BJ, Yang MB. Temperature induced gelation transition of a fumed silica/PEG shear thickening fluid. Rsc Advances. 2015:5(24):18367-18374.

8. Cwalina CD, Wagner NJ. Material properties of the shearthickened state in concentrated near hard-sphere colloidal dispersions. Journal of Rheology. 2014;58(4):949-967.

9. Jamali S, Boromand A, Wagner NJ, Maia J. Microstructure and rheology of soft to rigid shear-thickening colloidal suspensions. Journal of Rheology. 2015;59(6):1377-1395.

10. Ozel BG, Orum A, Yildiz M, Menceloglu YZ. Experimental study on the rheology of anisotropic, flocculated and low volume fraction colloids. Korea-Australia Rheology Journal. 2014;26(1):105-116.
11. Hoffman RL. Discontinuous and dilatant viscosity behavior in concentrated suspensions. II. Theory and experimental tests. Journal of Colloid and Interface Science. 1974;46(3):491-506.

12. Jiang W, Xuan S, Gong X. The role of shear in the transition from continuous shear thickening to discontinuous shear thickening. Applied Physics Letters. 2015;106:151902.

13. Montgomery DC. Design and Analysis of Experiments. New Jersey: John Wiley \& Sons, Inc; 2016.

14. Wu QM, Ruan JM, Huang BY, Zhou ZC, Zou JP. Rheological behavior of fumed silica suspension in polyethylene glycol. Journal of Central South University of Technology. 2006;13(1):1-5.

15. Haris A, Lee H, Tay TE, Tan VBC. Shear thickening fluid impregnated ballistic fabric composites for shock wave mitigation. International Journal of Impact Engineering. 2015;80:143-151.

16. Kang TJ, Kim CY, Hong KH. Rheological behavior of concentrated silica suspension and its application to soft armor. Journal of Applied Polymer Science. 2012;124(2):1534-1541.

17. Fahool M, Sabet AR. UV-visible assessment of hydrocluster formation and rheological behaviour in bimodal and mono-disperse shear thickening fluids. Rheologica Acta. 2015;54(1):77-83

18. Hasanzadeh M, Mottaghitalab V, Rezaei M. Rheological and viscoelastic behavior of concentrated colloidal suspensions of silica nanoparticles: A response surface methodology approach. Advanced Powder Technology. 2015;26(6):15701577.

19. Warren J, Offenberger S, Toghiani H, Pittman Junior CU, Lacy TE, Kundu S. Effect of Temperature on the Shear-Thickening Behavior of Fumed Silica Suspensions. Acs Applied Materials \& Interfaces. 2015;7(33):18650-61.

20. Baharvandi HR, Khaksari P, Kordani N, Alebouyeh M, Alizadeh M, Khojasteh J. Analyzing the Quasi-static Puncture Resistance Performance of Shear Thickening Fluid Enhanced P-aramid Composite. Fibers and Polymers. 2014;15(10):2193-2200.

21. Jiang T, Zukoski C. Rheology of high density glass of binary colloidal mixtures in unentangled polymer melts. Soft Matter. 2013;9(11):3117-3130 